\title{
NOVEL CHATTERBOT SYSTEM OF ESTIMATING CURRENT USER INTERESTS BY MEANS OF WEB INFORMATION
}

\author{
Miki Ueno, Naoki Mori, Keinosuke Matsumoto \\ Department of Computer and Systems Sciences, Graduate school of Engineering, Osaka Prefecture University \\ 1-1 Gakuencho, Nakaku, Sakai, Osaka 599-8531, Japan \\ e-mail: ueno@ss.cs.osakafu-u.ac.jp,mori@cs.osakafu-u.ac.jp, matsu@cs.osakafu-u.ac.jp \\ http://zope.ss.cs.osakafu-u.ac.jp/
}

\begin{abstract}
Human like conversation systems are one of the most important target of computer engineering. To achieve natural conversation, estimating current user interests is the essential issue. In this paper, we proposed the novel chatterbot which can estimate current user interests by means of Web information to solve above problem. In proposed chatterbot, interests are represented by interest vectors that were created by Bulletin Board System (BBS) data. To show the effectiveness of the proposed method, the computational experiments are carried out taking several BBS data as examples.
\end{abstract}

Keywords: chatterbot, estimating current user's interest, web information.

\section{INTRODUCTION}

Attempting to create an intelligent conversation system [1-4] is one of the most important and interesting themes in computer engineering. However, approaches that use natural language processing and artificial intelligence techniques have not yet been able to create an enjoyable experience for users.

In conversation systems, one of the most important issue is how to estimate current user interests $[5,6]$. In this paper, we propose the novel chatterbot that estimates user interests by using Web information [7]. In this chatterbot, interests are represented by interest vectors that are created by Bulletin Board System (BBS) called "2channel" (2ch) [8]. Although lots of interest vectors can be obtained by our method, we have not shown the generality of interest vectors of $2 \mathrm{ch}$ for various user inputs. We show the effectiveness and generality of interest vectors of proposed chatterbot by computer simulation taking several BBS data as pseudo user inputs.

We first present the constitution of the proposed chatterbot in section 2. We propose a novel chatterbot in section 3. Computer experiments are described in section 4 . We proposed new method to estimate current user's interests in section 5. In section 6, we had computer experiments to confirm effectiveness of our method, while section 7 introduces possible applications of the proposed chatterbot. Finally, in section 8 , we conclude this study.

\section{CONSTITUTION OF PROPOSED CHATTERBOT}

In this study, the proposed chatterbot is constituted in the following manner.

\section{Interpretation}

The chatterbot receives an input sentence and formats this input for the following processes. The first step of interpretation is morphological analysis of the input. Here, we utilize Sen [9], which is one of the leading pure Java morphological analysis libraries for Japanese.

\section{Replying}

The chatterbot replies to the user with an appropriate expression. We adopt short-term memory for reasonable conversation and a user logging system for retrieving information on the specific user. The proposed chatterbot also checks Wikipedia [10] to formulate an appropriate response to unknown keywords.

\section{Learning}

The chatterbot can memorize new statements and revise its memory. If the chatterbot fails to supply an appropriate response using its memory, it will ask the user about the user's input sentence or any 
unknown keywords. The user can teach the chatterbot the meaning of new words, or force the chatterbot to forget a specific part of its memory.

\section{Personality}

Since the chatterbot matures through conversation with users, the personality of the chatterbot is crucial for forging an emotional bond between the users and the chatterbot. The proposed chatterbot maintains a self-portrait and introduces unique topics of conversation.

\section{UTILIZING WEB INFORMATION}

\subsection{BASIC CONCEPT}

We propose a novel chatterbot that uses Web information. A simple approach to utilize Web information is to extract the sentences from Wikipedia [10] or from search engine results. In this study, we propose a novel method for making the appropriate replies with estimating the current user interests. In this paper, we focus on the estimating current user interests and do not show the detail of making replies. Since estimating the user interests is very important to achieve interesting conversation, this study is essential to construct our chatterbot.

We use the data of Bulletin Board System (BBS) to estimate user interests because BBS data is already categorized into typical fields.

\subsection{TARGET BBS}

We selected 2ch [8] as the target BBS because $2 \mathrm{ch}$ is the most comprehensive forum in Japan and covers diverse fields of interest. The top level unit is called "category". Several boards belong to a "category". It has more than 600 active boards including "Social News", "Computers", and "Cooking". Each board usually has many active threads that have main topics for discussion.

\subsection{UTILIZING BBS INFORMATION}

The following 2 approaches are adopted to use the information from the BBS.

Approach1: If the chatterbot knows the user's interests before the session, the chatterbot will try to use words that appear frequently on the board related to the user's interests.

Approach2: The chatterbot determines the user's interests automatically by using statistical information from the user's conversation log.

\subsection{DISTANCE BETWEEN BOARDS}

\subsubsection{DEFINITION OF DISTANCE}

In this study, we define the distance between two boards in the 2ch BBS as a simple Euclidean distance. If the similarity of two boards is high, the distance between those 2 boards will be low.

The words set throughout the entire BBS is defined as $W$, and the $i$-th word is denoted as $w_{i}$. We set $|W|=M$. Then the feature vectors of board $x$ and $y, \hat{\mathbf{x}}$ and $\hat{\mathbf{y}}$ respectively, are defined as follows.

$x=\left(\begin{array}{c}N_{w_{1}}^{x} \\ \vdots \\ N_{w_{i}}^{x} \\ \vdots \\ N_{w_{M}}^{x}\end{array}\right), y=\left(\begin{array}{c}N_{w_{1}}^{y} \\ \vdots \\ N_{w_{i}}^{y} \\ \vdots \\ N_{w_{M}}^{y}\end{array}\right)$

$N x, w_{i}$ :The number of words $w_{i}$ in board $x$

$N y, w_{i}$ :The number of words $w_{i}$ in board $y$

$$
\hat{\mathbf{x}}=\frac{\mathbf{x}}{|\mathbf{x}|}, \hat{\mathbf{y}}=\frac{\mathbf{y}}{|\mathbf{y}|}
$$

Then, the distance between boards $x$ and $y$ is defined as follows.

$$
D(x, y)=\sqrt{\sum_{i=1}^{M}\left(\hat{x}_{i}-\hat{y}_{i}\right)^{2}}
$$

\subsubsection{STOP WORDS REDUCTION}

We aim to find the typical relationship between words and the particular board. Therefore extremely common words such as "I" or "you" are not suitable for this purpose. To solve this problem, words which appear in $N$ boards are regarded as stop words and removed, where $N$ is the number obtained by multiplying the total number of boards and parameter $\gamma$. We also removed "user name", "date and time" and inappropriate no good words by pattern matching.

\subsection{ALGORITHM OF SIMPLE REPLYING}

We show the algorithm of simple replying as following. To confirm the efficiency of using user's interest, we performed simple experiment based on this algorithm in Section 4.

[Simple Replying]:

1. Save all sentences on board $i$ from the Web to set $S_{i}$.

2. Let the number of all boards from which sentences are saved be $n$. Define $S=\bigcup n, i=1 S_{i}(\neq \varphi)$ as the total set for $n$ boards.

3. Set the probability of using the positive set $p$.

4. Let the first noun in the sentence be $k$. Set the positive set as 
$S^{\text {true }}=\{x \mid x \in S, x$ is the sentence which

contains word $k$.

Let $S^{\text {false }}=S \backslash S^{\text {true }}$.

5. Select a sentence randomly from $S^{\text {true }}$ with probability $p$, or from $S^{\text {false }}$ with probability of $1-p$, and output this sentence after the formatting procedure. If $S^{\text {true }}=\varphi$ or $S^{\text {false }}=\varphi$, a sentence is selected from $S$.

\section{COMPUTER EXPERIMENTS 1}

We evaluate whether the board's distance can be practically used to detect user interests. This experiments shows the effectiveness of Approach1 in 3.3.

\subsection{EXPERIMENTAL SETUP AND RESULTS}

In this study, we selected $2 \mathrm{ch}$ [8] for the computer experiments because it is one of the largest BBSs in Japan and its BBS topics are categorized well (Top $\rightarrow$ Category $\rightarrow$ Board $\rightarrow$ Thread). First, we selected five boards: Math, Physics, Literature, Beauty (topics about cosmetics), and Jobs (topics about employment). We only focus on nouns when analyzing target words. Replying of chatterbot is generated by algorithm shown in $\mathbf{3 . 5}$.

Table 1 shows the top 10 most frequently used words on each board. Table 2 shows the distance between each pair of boards.

Table 1. Top 10 words on each board $(\gamma=0.6)$

\begin{tabular}{|c|c|c|c|c|}
\hline Mathematics & Physics & Literature & Beauty & Jobs \\
\hline mathematics & time & smile & face & $\begin{array}{l}\text { informal } \\
\text { decision }\end{array}$ \\
\hline proof & existence & novel & male & company \\
\hline book & physics & $\begin{array}{c}\text { work of } \\
\text { art }\end{array}$ & female & enterprise \\
\hline definition & light & book & eye & work \\
\hline 1 & universe & novelist & hair & interview \\
\hline number & understanding & literature & skin & university \\
\hline understanding & explanation & \begin{tabular}{|l|} 
love \\
\end{tabular} & love & age \\
\hline \begin{tabular}{|c|} 
case \\
\end{tabular} & human & human & nose & activity \\
\hline existence & earth & Japan & beautiful & recruit \\
\hline you & case & age & effect & day \\
\hline
\end{tabular}

Table 2. Distance between boards in 2ch BBS $(\gamma=0.6)$

\begin{tabular}{|c|c|c|c|c|c|c|}
\hline & Math & Physics & Literature & Beauty & Jobs & Norm \\
\hline Math & 0 & 0.87 & 1.04 & 1.18 & 1.12 & 26975.91 \\
\hline Physics & 0.87 & 0 & 1.02 & 1.17 & 1.13 & 28850.04 \\
\hline Literature & 1.04 & 1.02 & 0 & 1.03 & 1.06 & 37687.74 \\
\hline Beauty & 1.18 & 1.17 & 1.03 & 0 & 1.10 & 34642.16 \\
\hline Jobs & 1.12 & 1.13 & 1.06 & 1.10 & 0 & 31950.71 \\
\hline
\end{tabular} Note: rounded to two decimal places

To investigate the effects of applying BBS information to determine user interests, we conducted the following questionnaire survey of users. Specifically, we evaluated the extent to which the conversational quality of the proposed chatterbot improved. The procedure for the experiment is presented below. We set the number of user inputs of each trial to 10 times.

\section{BBS Experiment}

1. The target user converses with the original chatterbot freely without BBS Information.

2. The target user then selects one board of interest. In this step, we set $n=1$ in replying algorithm in $\mathbf{3 . 5}$ and set selected board as $S_{1}$.

3. We adjust the chatterbot settings to use sentences from the board selected in Step 2.

4. The target user converses with the chatterbot that is using board information.

5. Repeat Step 1 - Step 4 five times and count the number of trials that user could be satisfied the chatterbot outputs from the view point of their interests in conversations of Step 1 and Step 4.

The results of the questionnaire survey are shown in Table 3, where the words after user name (Math, Fashion, TV Game) were user's interests. $n / 5$ represents that user could be satisfied chatterbot outputs $n$ times in 5 trials of BBS Experiment.

Table 3. Evaluation of chatterbots before and after utilization of BBS information

\begin{tabular}{|c|c|c|}
\hline & No BBS Info & Use BBS Info \\
\hline User1(Math) & $1 / 5$ & $5 / 5$ \\
\hline User2(Fashion) & $2 / 5$ & $4 / 5$ \\
\hline User3(TV Game) & $2 / 5$ & $4 / 5$ \\
\hline
\end{tabular}

\subsection{DISCUSSION}

The results shown in Table 2 indicate that the distance between Math and Physics is smaller than the distance between Math and Beauty. Since Math and Physics have several top 10 words in common in Table 1, it is natural that the distance between Math and Physics is small. On the other hand, since Math and Beauty have no words in common in Table 1 the distance between Math and Beauty is large. The results in Table 1 and Table 2 are reasonable, and appropriate values were obtained by checking the similarity of the different boards.

Table 3 shows that utilizing board information is effective for satisfying the user's interests.

\section{ESTIMATING CURRENT USER INTERESTS}

We proposed method and showed effectiveness of Approach1 in $\mathbf{3 . 3}$ by Experiment 1 shown in 
section 4. However, Approach2 in $\mathbf{3 . 3}$ is more important for chatterbot system.

In this section, we proposed method of estimating user interests automatically by user's conversational $\log$ as Approach2 in 3.3.

Generally, user interests are changing during conversation. If the chatterbot uses all logs equality, it is difficult to estimate current topics. To solve this problem, we propose the following method which estimate current user's interests by emphasizing the current user input.

The proposed chatterbot has a user $a$ 's interest vector $c^{a}$ which represents user's internal state. Each vector element of $\boldsymbol{c}^{a}(0 \leq \boldsymbol{c} a, j \leq 1)$ relates to category $j$ of stored BBS board information. If the degree of user interests in related board is maximum, the value of vector element becomes 1 . On the other hand, use has no interest in that board, the value becomes 0 .

The proposed chatterbot tries to answer based on the topic of board of larger $\boldsymbol{c}^{a, j}$. However, $\boldsymbol{c}^{a}$ is made by all logs, there is problem that the proposed chatterbot fails to understand current topics. To avoid this, the proposed chatterbot decides the topic of answer as follows:

We define the latest input in time step $T$ as $S_{T}$. Old inputs are represented by $S_{T-1}, S_{T-2}, \ldots, S_{1}$. We also define the changing topic vector $\sigma_{S_{T}}$ as follows:

$$
\left\{\boldsymbol{y}_{S_{T}}\right\}_{j}=\sum_{i=1}^{W} N_{i} R_{i}^{j}
$$

where $N_{i}$ is the frequency of word ${ }_{i}$ in $S_{T}$ and $R_{i}^{j}$ is the frequency word $i$ in board $j$.

Next, the topic vector $\boldsymbol{t}^{\alpha}$ which $i$-th element represents the current significance of board $i$ is defined.

We set the default value of all elements of $\boldsymbol{t}^{\alpha}$ to 0 . The proposed chatterbot calculates $\boldsymbol{t}^{\alpha}$ as follows whenever the proposed chatterbot obtains the newest input $S_{T}$.

$$
\boldsymbol{t}^{\alpha}=\sum_{i=1}^{T} \gamma^{i-1} \boldsymbol{y}_{S_{T-i+1}}
$$

where $\gamma, 0 \leqq \gamma \leqq 1$, is the discount rate. As $\gamma$ decreases, the influence of past inputs decreases. We define normalized unit vector of $\boldsymbol{t}^{\alpha}$ as $\hat{x}^{a} \hat{t}^{a}$.

Since we assume that user interests are constant in short term, we consider that the weight of past inputs and that of current inputs are the same.
Therefore $\boldsymbol{c}^{\alpha}$ is obtained by $\boldsymbol{t}_{\gamma=1}^{\alpha}$ for $\gamma=1$ as follows:

$$
\mathbf{t}_{\gamma=1}^{\alpha}=\sum_{i=1}^{T} \sigma_{S_{T-i+1}}, \mathbf{c}^{a}=\hat{\mathbf{t}}_{\gamma=1}^{a}
$$

The proposed chatterbot decides the board to use by referring to $c^{\alpha}$ and $\hat{t}^{a}$. We define board deciding vector $\boldsymbol{b}$ as follows:

$$
\mathbf{b}=\eta \mathbf{c}^{a}+(1-\eta) \hat{\mathbf{t}}^{a}
$$

where $0 \leq \eta \leq 1$. The proposed chatterbot selects board $i$ in proportion to $\boldsymbol{b}_{i}$.

\section{COMPUTER EXPERIMENT 2}

\subsection{EXPERIMENT 2-1}

To evaluate the algorithm for estimating user's in-terest, we did a statistical experiment using texts of boards from $2 \mathrm{ch}$ which are already categorized.

In this section, we performed computer experiments in order to confirm our method of estimating user's interest based on the distance of boards shown in section $\mathbf{5}$.

To prepare the experiment, we applied the follow-ing processing to $2 \mathrm{ch}$ data.

1. Combine continuous nouns into one word.

2. Remove stop-words from test data.

We would like to obtained statistical results, lots of user inputs of a certain interests are required.

However, to get such a large data is difficult, we utilized randomly selected sentences from a board of $2 \mathrm{ch}$ as pseudo user inputs. We regarded the category of selected board as the interests of those inputs.

\subsubsection{SETTING OF EXPERIMENT}

1. Select 3 boards "Math", "Physics" and "Beauty" from 2ch for target boards.

2. The experiment is carried out in following 3 combination of board A and board B.

3. These 3 patterns are selected based on the result of Table 2 .

(a) board A: Math, board B: Physics The example of distance between boards is small.

(b) board A: Math, board B: Beauty The example of distance between boards is large.

(c) board A: Beauty, board B: Math Same pairs of boards as (b), but board A and B is opposite order. 
4. Top 10,000 frequent words of board $\mathrm{A}$ and board B on Jun. 23rd, 2009 are defined as the group of words for estimating user interest. These words are the same as words obtained in experiment 1 in section 4 .

5. Text data of board A and board B posted from Nov. 10th, 2012 to Nov. 16th, 2012 were separated into sentences and put each boards' sentences into $\mathrm{U}_{\mathrm{A}}$ and $\mathrm{U}_{\mathrm{B}}$ respectively. Those sentences are used as pseudo user inputs in the following experiment. We select $n$ sentences from $\mathrm{U}_{\mathrm{A}}$ and $m$ sentences from $\mathrm{U}_{\mathrm{B}}$ randomly. In experiment, $n$ sentences from $\mathrm{U}_{\mathrm{A}}$ are used first and then $m$ sentences from $\mathrm{U}_{\mathrm{B}}$ are used after finishing $\mathrm{U}_{\mathrm{A}}$ sentences. Finally, $n+m$ inputs are evaluated.

6. Each element of board define vector $\boldsymbol{b}$ in Eq. (4) shows the level of interests of the corresponding board. In this experiment, board define vector has two elements corresponding to board A and board B. The variation of each element is observed in order to check the tendency of $2 \mathrm{ch}$ boards and definition of boards define vector.

Table 4 displays conditions of experiment. We set $\eta=0.2$. $\eta$ is the parameter controlling the sensitivity of latest input. 100 results of board define vector of different random seeds are obtained.

Table 4. Experimental conditions

\begin{tabular}{|l|l|}
\hline $\begin{array}{c}\text { Number of sentences sampled } \\
\text { from board A }: n\end{array}$ & 10 \\
\hline $\begin{array}{l}\text { Number of sentences sampled } \\
\text { from board B }: m\end{array}$ & 10 \\
\hline $\begin{array}{l}\text { Number of sentences per log }: n \\
+m\end{array}$ & 20 \\
\hline Times & 100 \\
\hline Coefficient $\eta$ & 0.2 \\
\hline Discount rate $\gamma$ & 0.2 \\
\hline
\end{tabular}

To make the effectiveness of board define vector $\boldsymbol{b}$ in Eq. (4) clear, we did a t-test(two-sided test) every step between 100 trials of the first element of $\boldsymbol{b}$ as interest level of board A and that of the second element of $\boldsymbol{b}$ as interest level of board B.

\subsubsection{CONSIDERATION AND RESULTS}

In this section, the $\mathrm{x}$-axis shows the input step and the y-axis shows the average of $\boldsymbol{b}$ element in 100 trials. First element of $\boldsymbol{b}$ represents the interest level of board A and Second element of $\boldsymbol{b}$ represents that of board B. In Figs.1-3, the source of pseudo input is changed from $U_{A}$ sentences to $U_{B}$ sentences on input step 11. Figs. 1-3 show the variation of level of interests of board A and that of board B are shown as 2 lines.

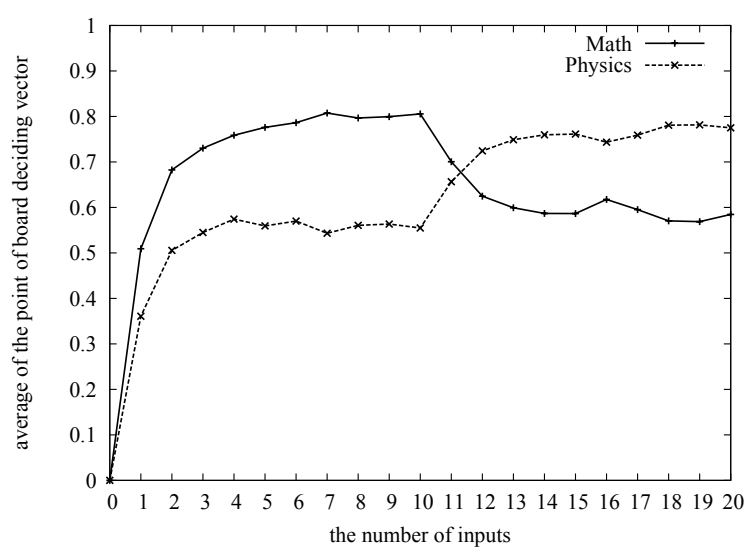

Fig. 1 - Experiment 2-1 (a) (board A: Math, board B:Physics)

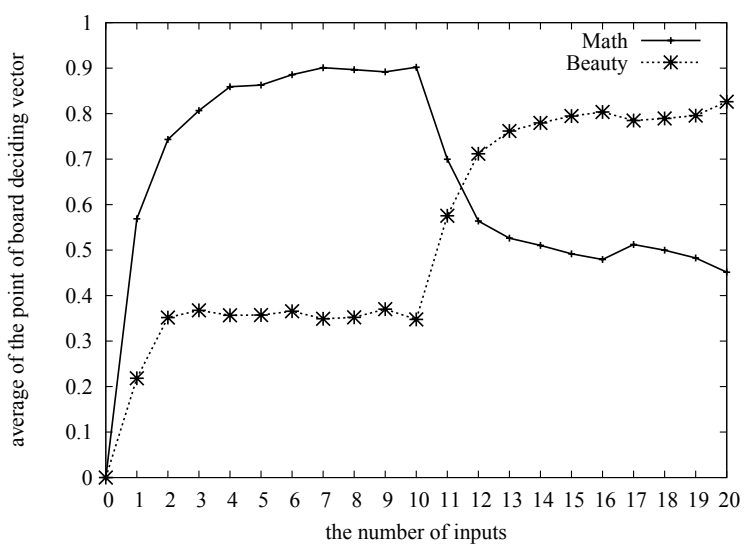

Fig. 2 - Experiment 2-1 (b) (board A: Math, board B: Beauty)

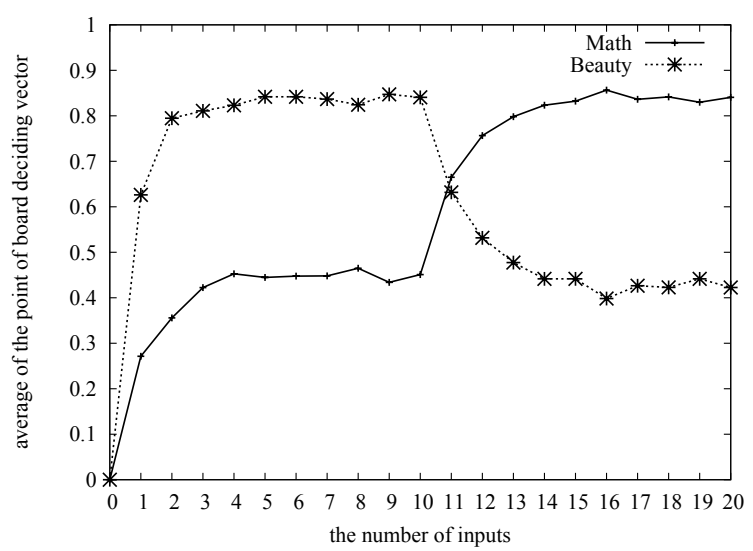

Fig. 3 - Experiment 2-1 (c)

(board A: Beauty, board B: Math)

(a) Math-Physics

Fig.1 shows the results of experiment 2-1 (a).

From input step 7 to input step 10, there exists significant difference at the significance level $1 \%$.

Value of interest level of board A gradually increases from step 1 to step 10 . On the other hand, from step 1 to step 6 and step 11 to step 20, there 
was no significant difference. Value of interest level of board B is increasing after input step 11 .

(b) Math-Beauty

Fig.2 shows the results of experiment 2-1 (b).

From step 1 to step 10 and from step 14 to 20, there exist significant difference at the significance level $1 \%$. In experiment 2-1 (a), significant difference is observed only 4 input steps $7,8,9$ and 10 , while significant difference is observed 17 input steps in experiment 2-1 (b). The difference between interests level of board A and that of board B of Fig. 2 is larger than that of Fig. 1.

This is because difference between math and beauty is larger than difference between math and physics. This result is reasonable because of result of Table 2 which shows the distance between math and beauty is larger than the distance between math and physics. The result of Table 1 also supports this results.

\section{(c) Beauty-Math}

Fig.3 shows the results of experiment 2-1 (c). In experiment 2-1 (b), first 10 inputs are selected from "Math" board next inputs are selected from "Beauty", while "Math" and "Beauty" is opposite order in experiment 2-1 (c). From step 1 to step 10 and from step 13 to step 20, there exists significant difference at the significance level $1 \%$. This result is the almost same as result in experiment 2-1 (b). Comparison result between Fig. 1 and Fig. 3 is similar that of Fig.1 and Fig.2, so the result is not affected by order of "Math" and "Beauty". This result show the maximum element of board define vector $\boldsymbol{b}$ represents user interests well.

\subsection{EXPERIMENT 2-2}

There exists small difference between the graph shape of Fig.2 and Fig.3. This is because the meaning of inputing "Math" sentences and "Beauty" sentences are different.

The results of experiment 2-1(b) and 2-1(c) show that the difference between values of interest level of "Math" and that of "Beauty" become large when input sentences is from "Math" board.

The increases of interest level of "Beauty" under using "Math" input is smaller than that of "Math" under using "Beauty" input.

We can make a following assumption by this fact.

\subsubsection{ASSUMPTION}

In board math, there are lots of specific words such as technical term. On the other hand, in beauty board, there are not so specific words.
The ratio of specific words such as technical term of "Math" board is larger than that of "Beauty".

\subsubsection{SETTING OF EXPERIMENT}

To investigate above assumption, we performed computer experiment under condition of setting $\eta=$ $0, \gamma=0, n=10, m=10$ in order to compare "Math" board and "Beauty" board on every step.

We checked error rate in following board setting:

(1) board A: Math, board B: Beauty

(2) board A: Beauty, board B: Math

Error rate of board $\mathrm{A}$ is the ratio of input steps that the interest level of board B is higher than that of board A even if input sentences are selected from board A. We calculated the error rate by using results of 100 trials in case of (1) and (2).

\subsubsection{RESULTS}

We obtained following results:

(1) Board A: Math, Board B: Beauty(using same sentences in experiment 2-1(b) )

Error rate of "Math" : 126(12.6\%)

Error rate of "Beauty" : 173(17.3\%)

(2)Board A: Beauty, Board B: Math(using same sentences in experiment 2-1(c))

Error rate of "Math" : 123(12.3\%)

Error rate of "Beauty" : 183(18.3\%)

The error rate of "Beauty" is always higher than that of "Math".

This results support that our assumption is true.

We may utilize this error rate for reliability of category of user interests. Lower error rate categories have high reliability in detecting user interests.

\section{APPLICATION}

We developed three types of applications for the proposed chatterbot. Since our chatterbot engine is independent part, we can easily apply the proposed chatterbot to other applications.

\subsection{STANDALONE APPLICATION}

This is a local application with a GUI [7]. Although it is possible to obtain Web information from Wikipedia or a BBS simultaneously via a network, this application also can be executed without a network by using a local dictionary. The GUI handles displaying characters, obtaining user input and printing responses of the chatterbot. Fig. 4 shows the look of our standalone chatterbot. 


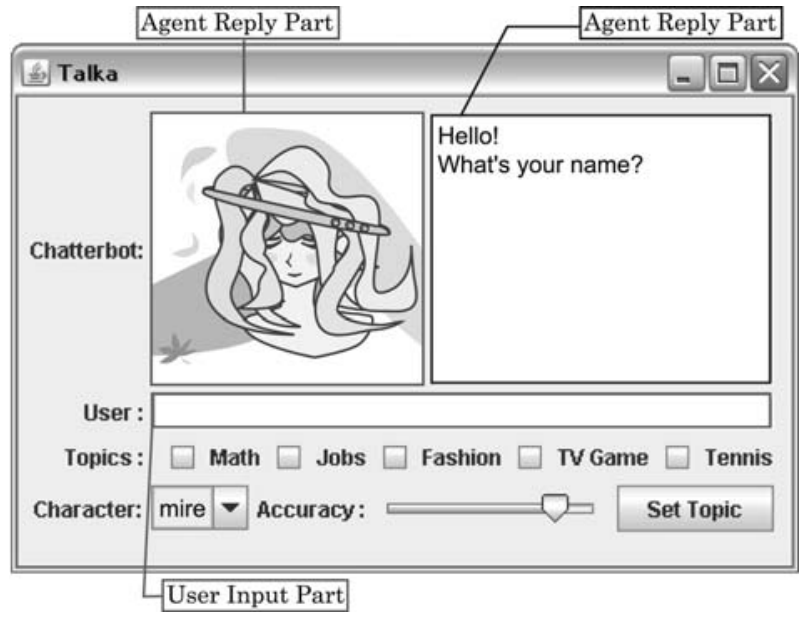

Fig. 4 - GUI of standalone chatterbot

\subsection{TWITTER BOT}

Twitter is a popular social network based communication service that enables its users to send, receive and view short messages known as tweets. Tweets are text-based posts of up to 140 characters that are displayed on the author's profile page and delivered to the author's subscribers, who are known as followers. We applied the proposed chatterbot as a Twitter bot using the Java-based Twitter API called Twitter4J. We have asked several testers to try our chatterbot in Twitter, and obtained positive opinions.

\subsection{PICTGENT}

We have proposed novel variation of our chatterbot by introducing picture information called "Picture Information Shared Conversation Agent" (Pictgent) [11] which can share picture information with user to create common topics of conversation easily. Pictgent can be applied to the field of eLearning.

The advantage of Pictgent becomes obvious when we apply it to education of children in single age because lots of children are bored with chatting by only text. Pictgent solves this problem by showing picture and realizing conversation with sympathy of user.

\section{CONCLUSION}

In this paper, we proposed the novel chatterbot that estimates user interests and showed the effectiveness and generality of interest vectors of proposed chatterbot.

The results of computer simulations taking several BBS data represented that proposed interest vectors can be applied for estimating general user inputs and follow the topic change immediately. We also showed the our proposed applications, such as
"Picture Information Shared Conversation Agent “ (Pictgent).

The following objectives will be studied in future research.

1. Making interest vectors of various fields.

2. Introducing proposed estimating user interest method into Pictgent.

3. Applying proposed chatterbot to human users.

4. Estimating not only user interests but user emotions from user inputs.

\section{ACKNOWLEDGMENTS}

This research was supported in part by a Grantin-Aid for Scientific Research (C), 22500208, 20102014 from the Ministry of Education, Culture, Sports, Science and Technology.

\section{REFERENCES}

[1] J. Weizenbaum, Eliza - a computer program for the study of natural language communication between man and machine, Communications of the ACM, (9), (1966).

[2] W. Chamberlain, The Policeman's Beard is Half Constructed, Warner Books, (1984).

[3] G. Guzeldere and S. Franchi, Dialogues with colorful personalities of early AI, Stanford Humanities Review, (4) 2 (1995).

[4] D. Vrajitoru, J. Ratkiewicz, Evolutionary sentence combination for chatterbots, International Conference on Artificial Intelligence and Applications (AIA 2004), ACTA Press, pp. 287-292, (2004).

[5] J. Kleinberg, Bursty and hierarchical structure in streams, Data Mining and Knowledge Discovery, vol. 7, 2003, pp. 373-397.

[6] T. Fujiki, T. Nanno, Y. Suzuki, and M. Okumura, Identification of bursts in a document stream, In First International Workshop on Knowledge Discovery in Data Streams, 2004, pp. 55-64.

[7] M. Ueno, N. Mori, K. Matsumoto, Novel chatterbot system utilizing web information for estimating current user interests, Proceedings of the 6th IEEE International Conference on IDAACS, Vol. 2, 2011, pp. 656-659.

[8] 2 channel, http://www.2ch.net/

[9] Sen, https://sen.dev.java.net/

[10] Wikipedia, http://wikipedia.org/

[11] M. Ueno, N. Mori, K. Matsumoto, Picture information shared conversation agent: pictgent, distributed computing and artificial intelligence advances in intelligent and soft computing, Springer, Vol. 151, 2012, pp. 9194. 


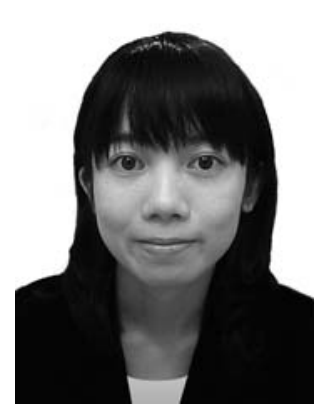

Miki Ueno, received her master degree of engineering from Osaka Prefecture University in 2012. She has been a student of Graduate School of Computer Science and Intelligent Systems, Osaka Prefecture University. Her research interests include dialogue system and affective engineering.

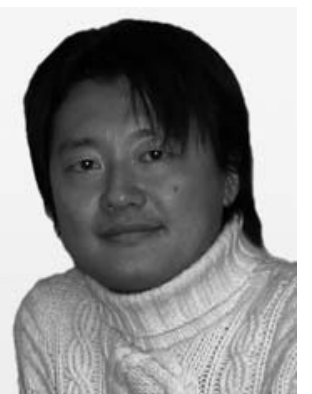

Naoki Mori, completed the M.E. and doctoral programs at Kyoto University in 1994 and 1997, and joined the faculty of Osaka Prefecture University as a research associate.

He is now an associate professor in the Department of Computer Science and Intelligent Systems at Osaka

Prefecture University. His research interests are genetic algorithms, artificial market and dialogue system. He received an ISCIE Paper Award in 1999. He holds a D.Eng. degree

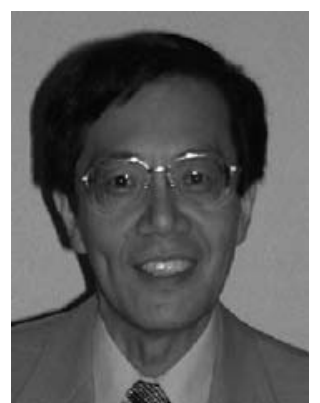

Keinosuke Matsumoto, is a professor in the Department of Computer Science and Intelligent Systems at Osaka Prefecture University. He received his B.S. and M.S. degrees in mechanics from Kyoto University in 1976 and 1978 respectively. He worked for Mitsubishi Electric Corporation as a researcher from 1978 to 1996 . He received his Ph.D. in electrical engineering from Kyoto University for his work on a knowledge-based approach to power system restoration. His research interests include software engineering, objectoriented technologies, and intelligent systems. 